\title{
Estudio de la cinética de oxidación de la superaleación MA 956
}

\author{
M.C. García-Alonso ${ }^{(*)}\left(^{* *}\right)$, J.L. González-Carrasco ${ }^{(*)}$ y M.L. Escudero ${ }^{(*)}$
}

Resumen Se estudia la cinética de oxidación de la superaleación MA 956 en el intervalo de temperaturas 800-1.200 ${ }^{\circ} \mathrm{C}$ durante tiempos de tratamiento de hasta $200 \mathrm{~h}$. Durante la oxidación, se forma una capa de alúmina fina, densa y muy bien adherida cuyo espesor aumenta al aumentar el tiempo y la temperatura. La cinética de oxidación puede describirse mediante una ley de tipo subparabólico. El crecimiento de la capa de alúmina en el intervalo de temperaturas estudiado parece estar controlado por dos mecanismos distintos; por encima de $1.050{ }^{\circ} \mathrm{C}$ se forma alúmina $\alpha$ y por debajo de los $900{ }^{\circ} \mathrm{C}$ alúmina $\gamma$.

Palabras clave: Cinética de oxidación. Superaleaciones ferríticas ODS. MA 956

\section{Study of the oxidation kinetics of the MA 956 superalloy}

\begin{abstract}
This work deals with the oxidation kinetics of the MA 956 superalloy in the temperature range of $800-1,200^{\circ} \mathrm{C}$ for up to $200 \mathrm{~h}$ exposure. During oxidation the alloy develops a fine, compact and very well adhered $\alpha$-alumina layer, the thickness of which increases with increasing time and temperature. The oxidation kinetics obeys a subparabolic type behaviour. The scale growth seems to occur by two different oxidation mechanisms; above $1,050{ }^{\circ} \mathrm{C}$, the oxidation process would be controlled by $\alpha$-alumina, and below $900^{\circ} \mathrm{C}$ by $\gamma$-alumina.
\end{abstract}

Keywords: Oxidation kinetics. ODS ferritic superalloy. MA 956.

\section{INTRODUCCIÓN}

La MA 956 es una superaleación con un alto interés tecnológico en aplicaciones a alta temperatura. Esta superaleación de base hierro lleva incorporado un dispersoide, la ytria, que le proporciona una fase termodinámicamente estable cuando es sometida a elevadas temperaturas (1). Dicho dispersoide mejora las propiedades mecánicas a alta temperatura (2) y facilita la formación de una capa de alúmina $\alpha$, fina, densa y muy bien adherida. La capa de alúmina proporciona un excelente comportamiento a la oxidación (3), por lo que esta aleación es muy apreciada en aplicaciones en las que la resistencia a la oxidación es el principal requisito.

Una de las propuestas más innovadoras para esta aleación ha sido la de su posible uso como biomate-

(*) Centro Nacional de Investigaciones Metalúrgicas, CENIM (CSIC), Avda. de Gregorio del Amo, 8. 28040Madrid (España).

(**) Ahora en el Institute for Advanced Materials, Joint Research Centre of the European Commission, 21020 Ispra (Va, Italia) rial para prótesis articulares (4 y 5). La propuesta se basa en que el material podría combinar la excelente biocompatibilidad y resistencia a la corrosión de la capa de alúmina con las buenas propiedades mecánicas del substrato metálico. Dado que la naturaleza de la capa de alúmina condicionaría su comportamiento in vivo, en este trabajo se estudia las condiciones óptimas de formación de una capa de alúmina químicamente inerte, como es la alúmina $\alpha$, densa, continua y bien adherida.

\section{PARTE EXPERIMENTAL}

La superaleación MA 956 es un material ferrítico con contenidos en masa de 4,5\% Al, 0,5\% Ti y $0,5 \% \mathrm{Y}_{2} \mathrm{O}_{3}$. La superaleación, suministrada por Inco Alloys, ha sido procesada por aleado mecánico y posteriormente sometida a laminación en caliente, sin posterior tratamiento de recristalización.

Para llevar a cabo el estudio de la cinética de oxidación se ha realizado una termogravimetría discontinua en el intervalo de temperaturas 800-1.200 ${ }^{\circ} \mathrm{C}$. El tiempo de tratamiento se varió de 1 a $200 \mathrm{~h}$. 
Los ensayos se llevaron a cabo con discos de $1 \mathrm{~mm}$ de espesor y $9,5 \mathrm{~mm}$ de diámetro, cortados perpendicularmente a la dirección de la barra. El acabado superficial consistió en un desbaste convencional seguido de un pulido en una suspensión de partículas de alúmina de $1 \mu \mathrm{m}$. Finalmente, todas las muestras se limpiaron con etanol. Las muestras así preparadas se depositaron en una navecilla de cerámica, introducida previamente en el horno durante 20 ó 30 min para conseguir la completa eliminación de agua absorbida. Transcurrido el tiempo de oxidación, las muestras se dejaron enfriar al aire. Las medidas de masa se realizaron en una balanza con una precisión de $\pm 10 \mu \mathrm{g}$.

\section{RESULTADOS Y DISCUSIÓN}

En la figura 1 aparece la variación de la ganancia de masa por unidad de área a lo largo del tiempo en el intervalo de temperaturas $800-1.200{ }^{\circ} \mathrm{C}$. A cortos tiempos de tratamiento, existe un gran aumento de la ganancia de masa, mayor a medida que aumenta la temperatura. Una vez transcurrido este primer período, la ganancia de masa es significativamente menor a medida que aumenta el tiempo de exposición. Esta disminución progresiva de la velocidad de oxidación indica la formación de un óxido compacto y bien adherido.

Según la teoría de Wagner (6), los sistemas sometidos a alta temperatura y controlados por procesos de difusión, a través de la red de la capa de óxido, siguen una cinética de oxidación descrita por una ecuación de tipo parabólico:

$$
(\Delta m / A)^{2}=k_{\mathrm{p}} t
$$

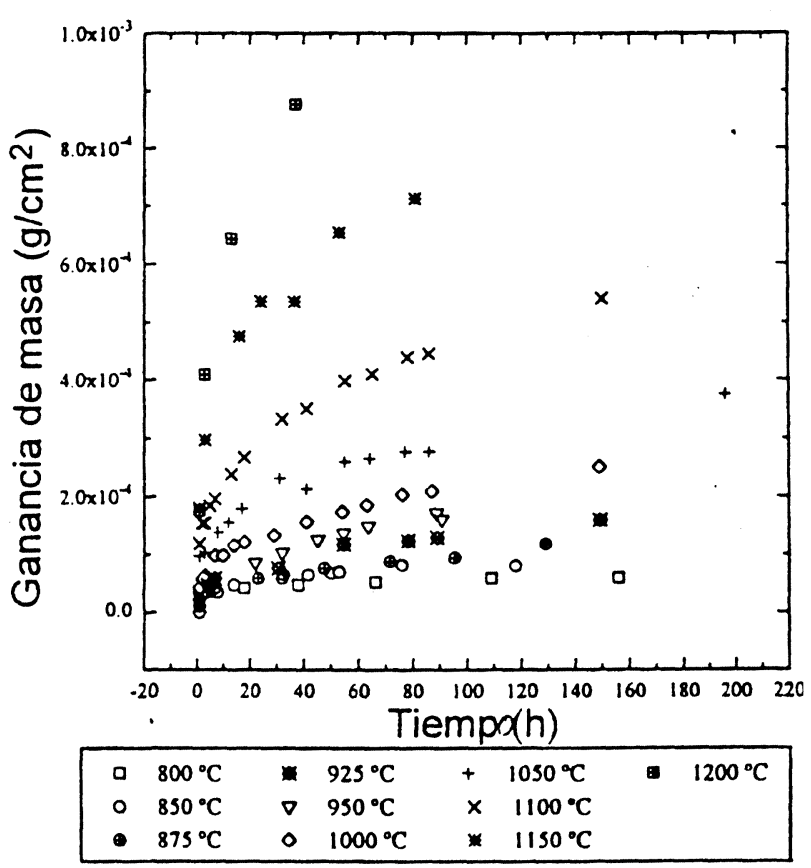

FIG. 1 siendo $\Delta m$ la ganancia de masa; $A$, el área expuesta; $k_{\mathrm{p}}$ la constante de velocidad parabólica y $t$, el tiempo de exposición. En determinados sistemas, es aconsejable la determinación de la constante de velocidad analizando la evolución con el tiempo del cuadrado de la variación de masa por unidad de superficie para cada tiempo de tratamiento $k_{\mathrm{p}}{ }^{\mathrm{i}}$ (7 y 8). Para ello, se deriva la ecuación [1] con respecto al tiempo:

$$
\mathrm{d}\left((\Delta \mathrm{m} / A)^{2}\right) / \mathrm{d} t=k_{\mathrm{p}}
$$

El valor de la constante instantánea de velocidad parabólica, $k_{\mathrm{p}}$ i , se ha representado en función del tiempo de tratamiento en la figura 2. En general, la constante es inicialmente más elevada, pero a medida que transcurre el tiempo de tratamiento va disminuyendo, hasta alcanzar un valor cuasiestacionario para largos tiempos de ensayo.

La desviación del comportamiento parabólico ideal, descrito por Wagner, puede deberse a aspectos tales como la formación y posterior transformación de alúminas metaestables (9-11) y a la variación del tamaño de grano de la capa de alúmina (12). A temperaturas bajas, y en las etapas más tempranas de la oxidación, crecen rápidamente lọs óxidos cúbicos metaestables, tipos alúmina $\gamma$ y $\delta$. Después, se transforman en alúmina $\theta$, de estructura monoclínica $y$, finalmente, tiene lugar una transformación lenta en alúmina $\alpha$, de estructura hexagonal. La velocidad de transformación de alúminas metaestables en otras más estables depende del tiempo y de la temperatura (13). Así, a temperaturas por encima de los $1.050^{\circ} \mathrm{C}$, la formación de alúmina $\alpha$ es prácticamente instantánea. La disminución de la constante de velocidad parabólica a temperaturas superiores a los $1.000{ }^{\circ} \mathrm{C}$

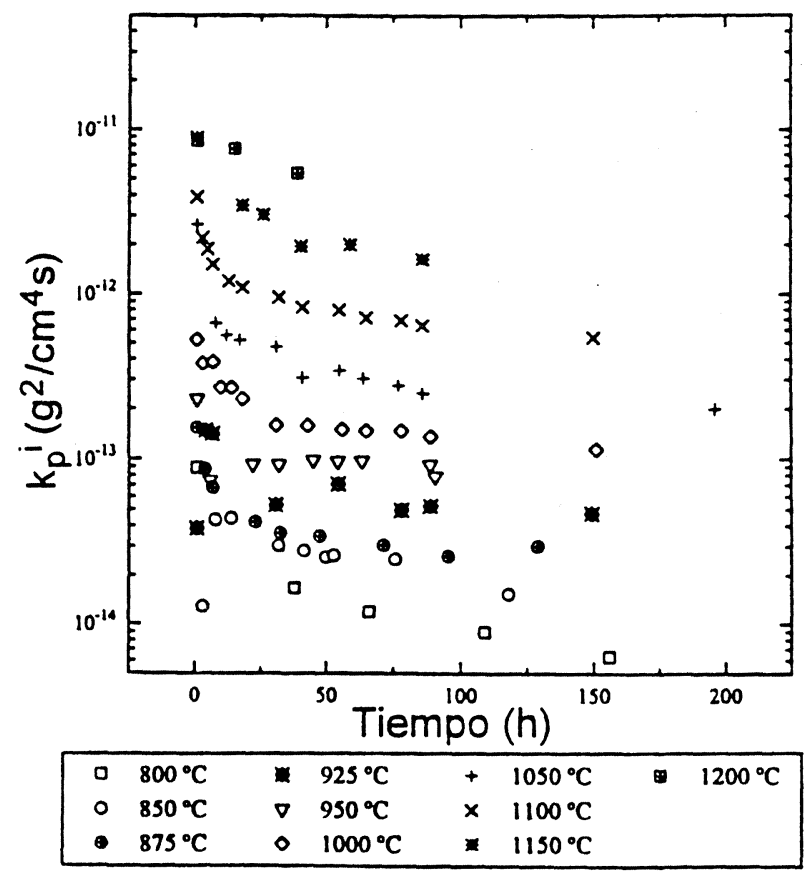

FIG. 2 
puede deberse a la variación del tamaño de grano de la capa de alúmina (9 y 12). Cuando el tamaño de grano es pequeño, es decir, en las primeras etapas de la oxidación, la difusión es más rápida que a tiempos largos, cuando el tamaño de grano es más grande.

Conocida la constante de velocidad parabólica para cada temperatura, se puede calcular la energía de activación de la reacción de oxidación a partir de la ecuación de Arrhenius, que relaciona $k_{\mathrm{p}}$ con la temperatura mediante la expresión:

$$
k_{\mathrm{p}}=k_{0} e^{(-Q / R T)}
$$

donde $Q$ es la energía de activación, $R$ es la constante de los gases, en julios o calorias, $k_{o}$ es la constante de velocidad a temperatura ambiente y $T$ es la temperatura absoluta.

En la figura 3 se representan los valores de la constante de velocidad en el estado cuasiestacionario (tiempos largos) frente al inverso de la temperatura. Dichos valores se han ajustado a dos rectas definidas una a altas y otra a bajas temperaturas. Dado que la pendiente de las rectas viene dada por la relación $Q / R$, se han determinado unas energías de activación de 89 y $42 \mathrm{kcal} / \mathrm{mol}$, que corresponden a un intervalo de temperaturas superior a 1.050 ${ }^{\circ} \mathrm{C}$ y por debajo de $900{ }^{\circ} \mathrm{C}$, respectivamente.

A partir del análisis del diagrama de Arrhenius (Fig. 3) se puede deducir la existencia de dos mecanismos de oxidación distintos, definidos por cada una de las rectas. Por debajo de los $900{ }^{\circ} \mathrm{C}$, la energía de activación obtenida indica que el proceso de oxidación está controlado por la formación de una alúmina metaestable tipo $\gamma(12)$. A partir de aproximadamente los $1.050^{\circ} \mathrm{C}$, la energía de activación obtenida corresponde a la formación de una alúmina estable tipo $\alpha$. (13 y 14). La región de transición se asocia a la formación de distintos óxidos, que, en este caso, se trataría de distintas fases metaestables de alúmina (15).

\section{CONCLUSIONES}

- Teniendo en cuenta la aplicación propuesta para esta superaleación, la formación de alúmina estable tipo $\alpha$ estaría asegurada a temperaturas por encima de los $1.050^{\circ} \mathrm{C}$. La evolución de las curvas de ganancia de masa indican la formación de una capa de alúmina bien adherida sin desprendimientos de capa.

- A temperaturas por debajo de $900^{\circ} \mathrm{C}$ el proceso de oxidación está controlado por la formación de una alúmina metaestable tipo $\gamma$.

- La variación de la constante instantánea de velocidad parabólica, $k_{\mathrm{p}}{ }^{\mathrm{i}}$, a lo largo del tiempo indica que la cinética de oxidación no sigue un comportamiento estrictamente parabólico.

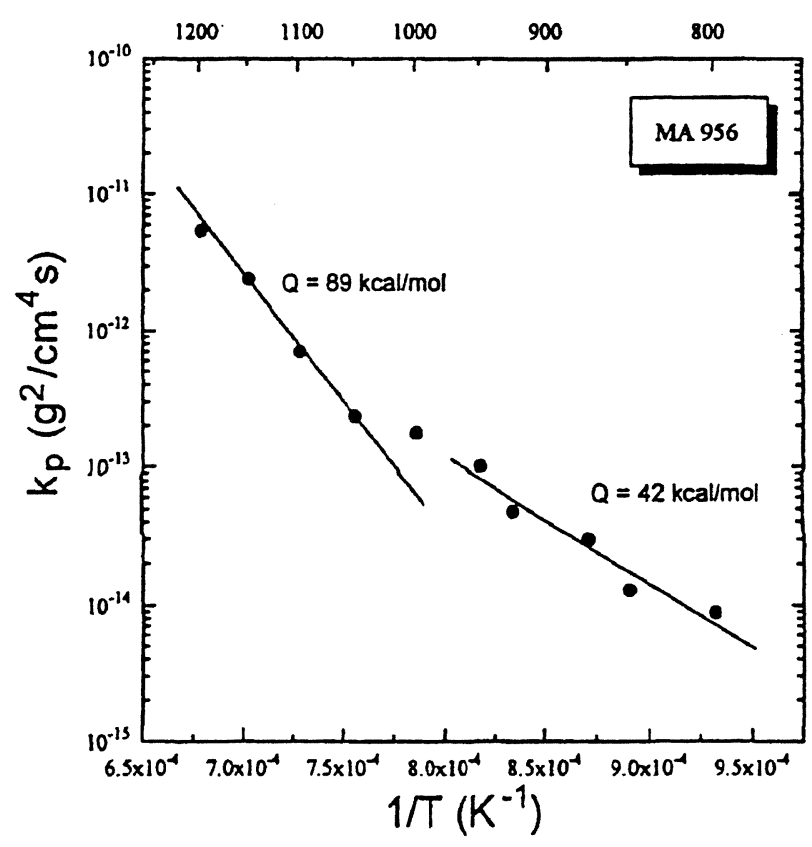

FIG. 3

\section{Agradecimiento}

Este trabajo ha sido financiado por la CICYT, proyecto MAT 95-0249-C03-01.

\section{REFERENCIAS}

(1) SmialeK, J.L. Metall. Trans. A, 22, 1991: 739

(2) Haghi, M. y Anand, L. Metall. Trans. A, 21, 1990: 353.

(3) Klug, R.C., Krauss, G., Matlock, D.K. y Saurez, F. Heat Resistant Materials (Proc. Conf.) Fontana, W.I (1991) 61

(4) Escudero, M.L. y GonZalez-Carrasco, J.L. Biomaterials, 15, 1994: 1.175.

(5) Escudero, M.L., Gonzalez-Carrasco, J.L., GarciaAlonso, M.C. y RAMIREZ, E. Biomaterials, 16, 1995: 735.

(6) WAGner, C. Atom. Movements. ASM, Metals Park, 1951: 153.

(7) Pieraggi, B. Oxid. Met., 27, 1987: 177.

(8) Brumm, M.W. y Grabke, H.J. Corros. Sci., 33, 1992: 1.677 .

(9) Garcia-Alonso, M.C. Tesis doctoral. Univ. Autónoma de Madrid. (1997).

(10) Moseley, P.T., Hyde, K.R., Bellamy, B.A. y Tappin, G. Corros. Sci., 24, 1984: 547.

(11) Pint, B.A., Martin, J.R. y Hobbs, L.W. Solid State Ionics, 78, 1995: 99.

(12) Quadakkers, W.J., Elschner, A., Speier, W. y Nickel, H. Appl. Surf. Sci., 52,1991: 271.

(13) Smialek, J.L. y Cibala, R. Metall. Trans. A, 14, 1983: 2.143 .

(14) Brumm, M.W. y Grabke, H.J. Corros. Sci., 33, 1992: 1.677 .

(15) Bennet, M.J. y Houlton, M.R. High Temperature Materials for Power Engineering. Ed. E. Bachelet, Kluwer Academic Publishers, Dordrecht (Holanda). 1990: 189.

(16) Ramanarayan, T.A., Ayer, R., Petkovic, R. y Leta, D.P. Oxid. Met.. 29, 1988: 445.

(17) Rybicki, G.C. y SMialeK, J.L. Oxid. Met. 31, 1989: 275. 